\title{
A Study on Integrated Continuous Varactor
}

\author{
Chun Wei Lin
}

\begin{abstract}
The continuous varactor is an adjustable storage element often used in filter, resonant and delay control circuit. This paper presents the method to implement linear integrated continuous varactor. Through driving several MOS capacitors into inversion-mode (IMOS) with different bias, various characteristics arise in the capacitance with respect to overdrive voltage. The IMOS with various capacitances can be further utilized to synthesize a wide range linear varactor. By using TSMC 0.35um CMOS process, the implemented varactor provides $\pm 33 \%$ tunable capacitance within $\pm 12 \%$ tuning range of voltage. In addition, the integrated varactor reaches picofarad scale which is considerably practical in many applications.
\end{abstract}

Keywords-varactor, resonant circuit, inversion mode, IMOS, tuning range.

\section{Introduction}

Capacitor is an electronic component that stores electric charge. Depending on its structure, conventional capacitor can be categorized as fixed capacitor, trimmer capacitor and variable capacitor. The fixed capacitor is made of 2 close plates separated by dielectric material. The construction of trimmer capacitor can be made by metal plates and the capacitance is variable according to the amount of overlap or distance between the two plates. A variable capacitor is a capacitor whose capacitance may be repeatedly changed mechanically or electronically. The mechanically controlled variable capacitor is similar to the construction of its less variant, the trimmer capacitor. The electronically controlled variable capacitor is also called integrated varactor and its capacitance is related to the thickness of the depletion layer of the $\mathrm{p} / \mathrm{n}$ junction in semiconductor transistor. Although the varactor can be designed with digitally tuned MOSFET capacitor, the capacitance and tunable range of single MOSFET capacitor is quite restricted. The discrete characteristic of capacitance and derivative switching power loss not only limits the application of digitally tuned varactor but also increases power consumption [1][2].

The linear integrated continuous varactor is often used for oscillators, filters and sensors. Particularly, the voltagecontrolled varactor is widely used in many adaptive control system. The traditional $\mathrm{D}=\mathrm{S}=\mathrm{B}$ structure of integrated varactor shown in Fig. 1(a) is simplest one of PMOS. As a result of $\mathrm{D}=\mathrm{S}=\mathrm{B}$ connected, the capacitance of varactor is varied with the voltage between bulk and gate. Within the situation of $V_{B}<V_{G}$ and $V_{B G}>\left|V_{T}\right|$, the transistor enters accumulation region and inversion region respectively that provides maximum capacitance [3][4]. The varactor behaves tunable property just in the depletion region when $V_{B}>V_{G}$ and $V_{B G}<\left|V_{T}\right|$.

Chun Wei Lin

Department of Electronic Engineering,

National Yunlin University of Science and Technology,

Douliou, Yunlin 64002, Taiwan,

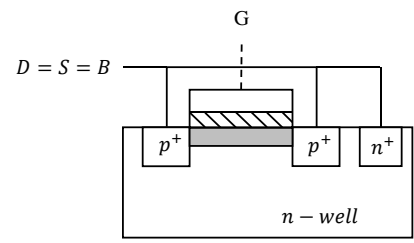

(a)

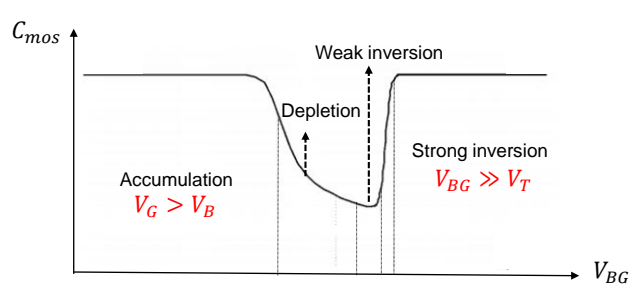

(b)

Figure 1 (a) the $\mathrm{D}=\mathrm{S}=\mathrm{B}$ structure of varactor, (b) the characteristic curve of $\mathrm{D}=\mathrm{S}=\mathrm{B}$ varactor

As shown in Fig. 1(b), the tunable region of $D=S=B$ varactor is limited within a small operation range. The accumulation-mode MOS (AMOS) structure shown in Fig. 2 was hence proposed to resolve this problem. The capacitance of AMOS structure can be effectively increased because the major carrier of MOS in accumulation-mode is electron having higher mobility than hole. However, to ensure that the MOS will stay in accumulation mode, the $p^{+}$ doping of PMOS is replaced by $n^{+}$to restrain electron holes from injecting into channel.

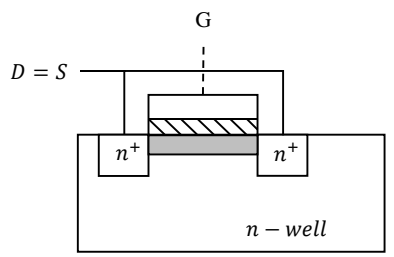

(a)

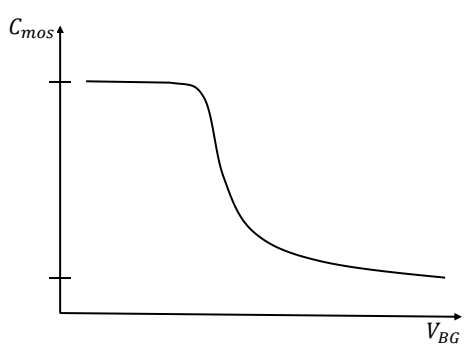

(b)

Figure 2 (a) the structure of AMOS varactor, (b) the characteristic curve of AMOS varactor.

Although The AMOS varactor provides wider tunable range, its application may be restricted by the required special semiconductor process. In case of using standard 
CMOS process, the inversion-mode MOS (IMOS) shown in Fig. 3 should be a convenient structure for implementation. In contrast to AMOS varactor, IMOS varactor should be drove into inversion region. The bulk is thus disconnected from drain and source to prevent the MOS gets into accumulation region.

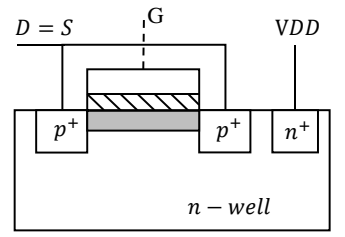

(a)

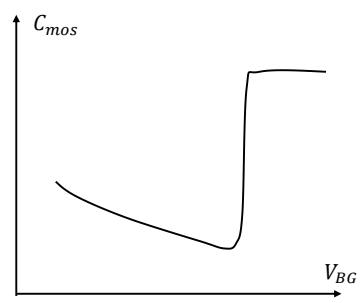

(b)

Figure 3 (a) the structure of IMOS varactor, (b) the characteristic curve of IMOS varactor.

\section{The proposed wide range IMOS varactor}

As the IMOS varactor is easy to be implemented by standard CMOS process, yet the confined tunable range and insufficient capacitance limits their utilization. We therefore present the method to implement the wide tunable range IMOS varactor which is very suitable for the adaptive control system. The concept of IMOS varactor is by utilizing the bulk and gate of MOS as plates of capacitor and the inversion layer acts as dielectric. To enter in inversion region, the IMOS varactor requires a bias, $\Delta v$, between source and gate of MOS as shown in Fig. 4. With the voltage variation between source and gate, $V_{\text {tune }}$, the inversion layer will be changed resulting in tunable capacitance [5]. That is, the inversion region appears in different range according to the value of bias voltage as shown in Fig. 5.

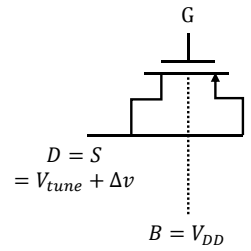

Figure 4 A simple p-type IMOS varactor

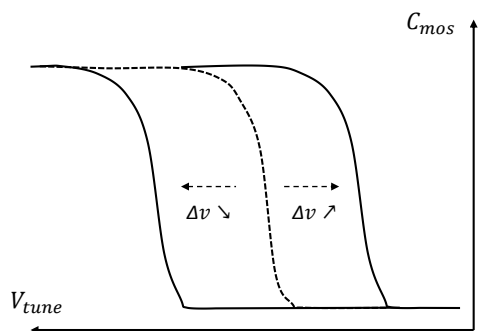

Figure 5 The characteristic curve of IMOS varactor.

The characteristic shift effect of IMOS varactor can be used to created different capacitance depending on given bias voltage. Fig. 6 shows the concept of this scheme. To simplify the complexity of implement, the gate is biased by a fixed voltage and the tuning voltage plus a little offset voltage is applied to the source of transistor. Through combining several IMOS varactors with different offset voltage, we can obtain a wide range continuous varactor. The more IMOS in parallel connection, the larger capacitance we have. The linearity and tuning range of varactor can be properly designed by choosing offset voltage. Choosing higher offset voltage, the tuning range is wider but the linearity is poor. On the contrary, the lower offset voltage brings better linearity within narrow tuning range as shown in Fig. 7.

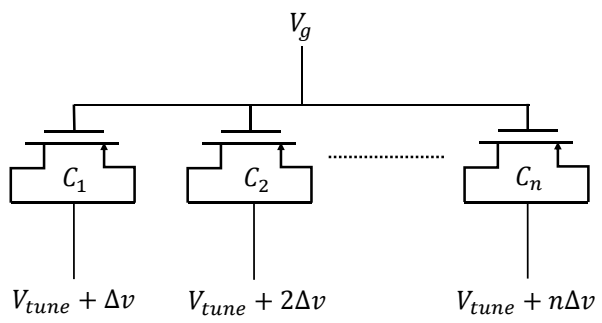

(a)

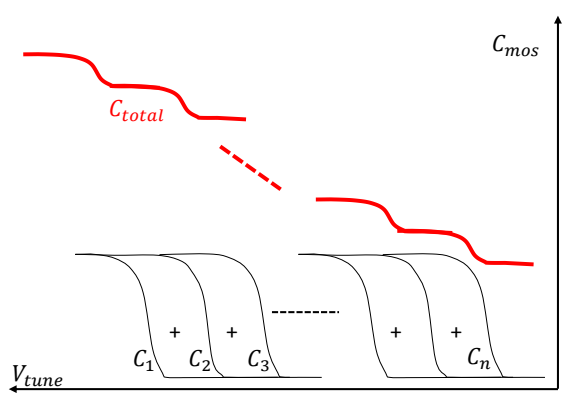

(b)

Figure 6 (a) the concept of wide range continuous IMOS varactor, (b) the characteristic curve of wide range continuous IMOS varactor.

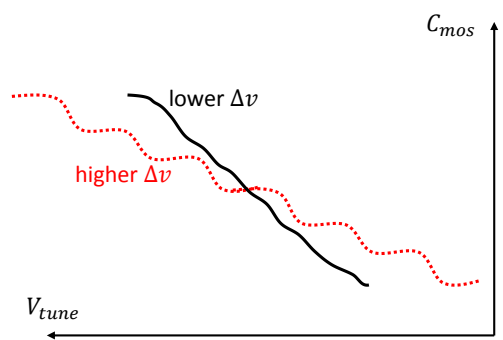

Figure 7 The capacitance of proposed varactor with lower and higher offset voltage.

\section{Application and implement of the proposed varactor}

To demonstrate the proposed method, we applied the integrated continuous varactor to the over temperature protection system of a Class D audio amplifier by using TSMC 0.35um CMOS process. Fig. 8(a) shows the 
architecture of Class D audio amplifier [6]. The over temperature protection system consists of the temperature sensor [7], the triangular wave generator and the varactor which Fig. 8(b), Fig. 8(c) and Fig. 8(d) shows their circuitry respectively.

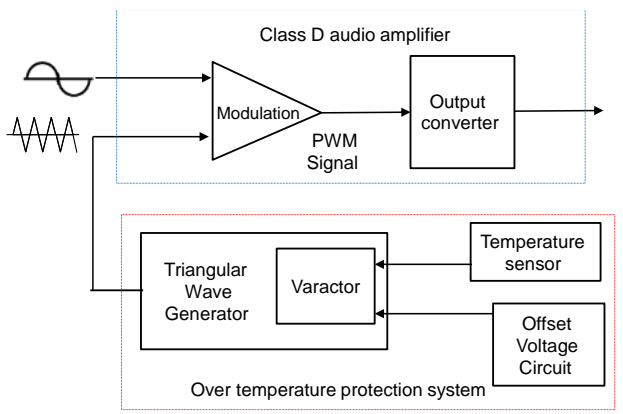

(a)

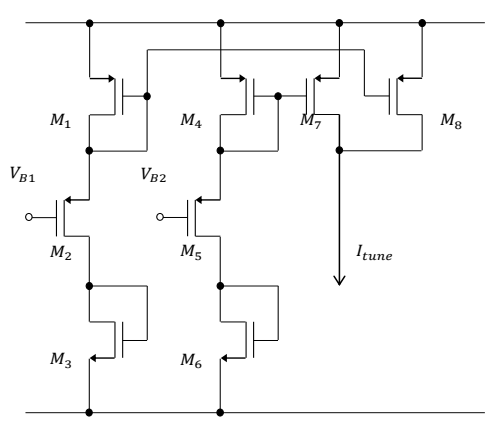

(b)

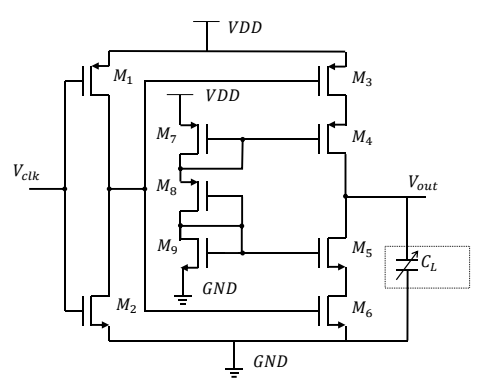

(c)

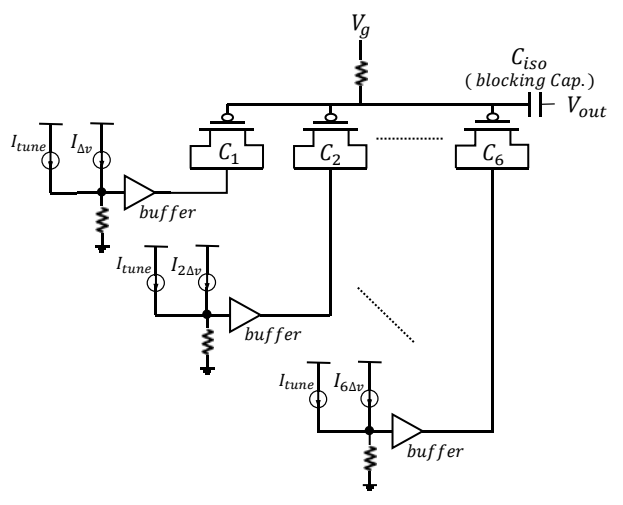

(d)

Figure 8 (a) the architecture of Class D audio amplifier [6], (b) the temperature sensor [7], (c) the triangular wave generator, (d) the continuous varactor.

Basically, this over temperature protect system is an adaptive temperature sensing loop. The linear temperature sensor as shown in Fig. 8(b) is embedded in audio amplifier to monitor heat dissipation of circuit. Once an over- temperature occurs in the system, the sensor produces linear corresponding current for varactor tuning. A conventional triangular wave generator as shown in Fig. 8(c) is simply an integrator. The output voltage on varactor will be charged or discharged in accordance with input clock signal. The resulted triangular wave is further applied to the modulator of audio amplifier which dynamically controls the power delivery. This adaptive protection loop prevents system from damage without shutting down system or bringing audio holes. The most important element of the adaptive protection loop is varactor as shown in Fig. 8(d). This integrated continuous varactor is composed of six IMOSs with all gates are connected to a reasonable bias voltage, $V_{g}=1.3 \mathrm{~V}$, and be isolated from output node of triangular wave by a blocking capacitor. The required offset voltages, $n \Delta v=0.1 n V$, are supplied by a reference current and its duplicated multiple, $I_{n \Delta v}$, passing through the resister. The corresponding current, I tune, from temperature sensor, further provides tuning voltage for varactor. The characteristic curves of six IMOSs in varactor is shown in Fig. 9(a). Within the tuning range, $2.4 \mathrm{~V}$ to $3.1 \mathrm{~V}$ (i.e. $2.7 \mathrm{~V} \pm 12 \%$ ), each IMOS gives tunable capacitance from $0.25 \mathrm{pF}$ to $2.25 \mathrm{pF}$. By summing up all IMOSs and taking into account blocking capacitor, the total tunable capacitance of varactor is from $1.8 \mathrm{pF}$ to $3.7 \mathrm{pF}$ (i.e. $2.7 \mathrm{pF} \pm$ $33 \%$ ) as shown in Fig. 9(b). The produced tunable capacitance enables the triangular wave generator to adjust the amplitude of its output signal from $1.65 \mathrm{~V}$ to $2.51 \mathrm{~V}$ (i.e. $52 \%$ increment); indeed, the power delivery of audio amplifier is reduced to almost half of maximum value.

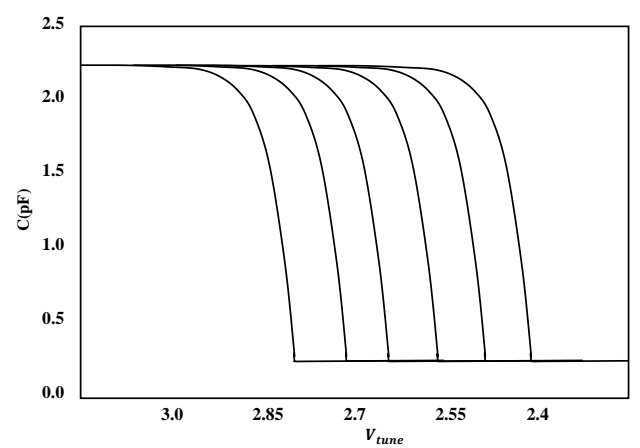

(a)

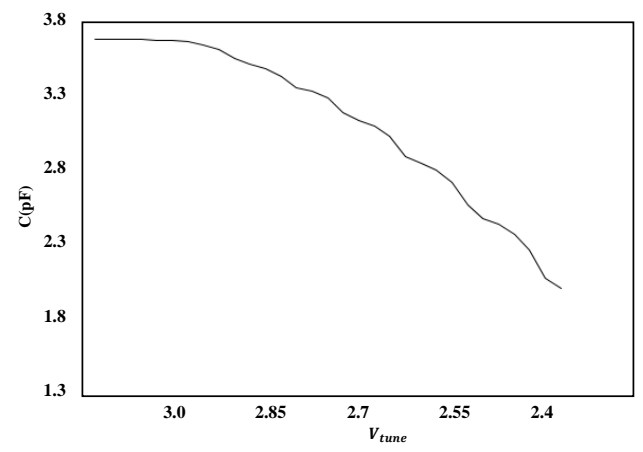

(b)

Figure 9 (a) the characteristic curves of six IMOSs, (b) the characteristic curves of varactor.

The Table I shows the comparison between implemented integrated continuous varactor and other prior works. The implemented varactor has better continuity as compared to 
digitally tuned varactor and provides wide tunable capacitance as well which is very suitable for the adaptive control system.

TABLE I. Comparison of several works

\begin{tabular}{|c|c|c|c|}
\hline & $\begin{array}{c}\text { Digitally tuned } \\
\text { varactor[2] }\end{array}$ & {$[8]$} & This work \\
\hline $\begin{array}{c}\text { Tunable } \\
\text { capacitance }\end{array}$ & $1.5 \sim 3.5 \mathrm{pF}$ & $25 \sim 50 \mathrm{fF}$ & $1.8 \sim 3.7 \mathrm{pF}$ \\
\hline Tuning range & $0.8 \mathrm{~V}$ & $0.61 \mathrm{~V}$ & $0.7 \mathrm{~V}$ \\
\hline Continuity & poor & good & good \\
\hline
\end{tabular}

\section{Conclusion}

In this paper, we have implemented a voltage-based integrated continuous varactor. By combining several IMOSs with different offset voltage, the wide tunable range varactor can be synthesized. The linearity and tuning range of varactor can be compromised to each other while choosing higher offset for wider tuning range and lower offset for better linearity. As a practical application, the implemented varactor have applied to the over temperature protection system of a Class D audio amplifier. Within $\pm 12 \%$ tuning range of voltage, the varacor has $\pm 33 \%$ tunable capacitance which enables flexible capability of power regulation.

\section{Acknowledgment}

The authors would like to thank National Chip Implementation Center (CIC) for technical support and chip fabrication.

\section{References}

[1] Lawrence Der, Stephen H. Lewis, Paul J. Hurst, "A SwitchedCapacitor Differencing Circuit with Common-Mode Rejection for Fully Differential Comparators," Proc. IEEE Midwest Symp. On Circuits and Systems, p.p. 911-914, 1993.

[2] C.W. Lin, R.F. Liu, "A Study on Temperature Protection for Class-D Amplifier," Proc. International Conference on Computer Science and Information Engineering, p.p. 108-113, 2015.

[3] P. Andreani, S. Mattisson, "On the use of MOS varactors in RF VCOs," IEEE J. Solid State Circuits, vol. 35, p.p. 905-910, 2000.

[4] R.L. Bunch, S. Raman, "Large-signal analysis of MOS varactors in CMOS -Gm LC VCOs," IEEE J. Solid-State Circuits, Vol. 38, p.p.1325-1332, 2003.

[5] J. Oehm and D. Pham-Stabner, "Linear controlled temperature independent varactor circuitry," Proc. IEEE The European Solid-State Circuits Conf., p.p. 143-146, 2002.

[6] C.W. Lin, B.S. Hsieh, "The Multilevel Technique for Improving Filterless Class-D Audio Amplifiers," Journal of Circuits, Systems and Computers, Vol. 23, No. 4, 1450047(19 pages), 2014.

[7] C.W. Lin, S.F. Lin, "A linear CMOS temperature sensor with an inaccuracy of $\pm 0.15^{\circ} \mathrm{C}$," IEICE Electronics Express Vol. 9, No. 20, pp. 1556-1561, 2012.

[8] A. Dec, H. Akima, K. Suyama, "A 5GHz LC VCO with extended linear-range varactor in purely digital 0.15 um CMOS Process," Proc. IEEE Radio Frequency Integrated Circuits Symposium, p.p.567-570, 2009.

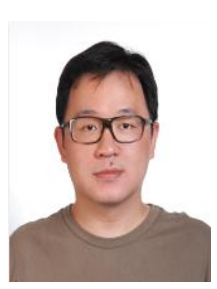

Chun-Wei Lin received BS degree in electronic engineering from Chung Yuan Christian University, Taiwan, R.O.C. From 2002, he was with SPIROX Corporation, working in the area of design and test mixed signal circuit. His research interests are in the area of integrated circuit design, VLSI testing, and computer aided design. He is a member of IEEE and IEICE. 